\title{
Foundation Document: uma solução para a elaboração de planos de manejo de unidades de conservação
}

\section{Michel Tadeu R. N. de Omena ${ }^{1}$, Jorge Altair Pinto Sturmer ${ }^{2}$, Paulo Santi Cardoso da Silva ${ }^{3}$ e Natalia Hanazaki ${ }^{4}$}

${ }^{1}$ Universidade Federal de Santa Catarina. Programa de Pós-Graduação em Ecologia. Campus Universitário. Trindade. Florianópolis-SC, Brasil (CEP 88040-900).

${ }^{2}$ Universidade Federal de Santa Catarina. Programa de Pós-Graduação em Administração. Campus Universitário. Trindade. Florianópolis-SC, Brasil (CEP 88040-900).

${ }^{3}$ Instituto Chico Mendes de Conservação da Biodiversidade. Av. Felicíssimo Rodrigues, 1542. Esquina. Urubici-SC, Brasil (CEP 88650-000).

${ }^{4}$ Universidade Federal de Santa Catarina. Laboratório de Ecologia Humana e Etnobotânica. Campus Universitário. Trindade. Florianópolis-SC, Brasil (CEP 88040-900).

Resumo. Este artigo propõe uma reflexão sobre a elaboração dos planos de manejo em áreas naturais protegidas no Brasil. Avaliamos a adaptação do Modelo Foundation Document, método utilizado pelo serviço de parques estadunidenses para elaboração de planos de manejo. Este método foi testado experimentalmente pelo órgão gestor de Unidades de Conservação federais em duas áreas, o Parque Nacional de São Joaquim (Santa Catarina) e a Reserva Extrativista Marinha de Soure (Pará). As observações descritas neste artigo são fruto da participação na elaboração do Plano de Manejo do Parque Nacional de São Joaquim, no período de 2016 a 2018. A utilização do Método Foundation Document mostrou-se vantajosa em relação ao modelo tradicional de elaboração de planos quanto ao tempo de conclusão do documento, ao tamanho, ao prazo de validade, à facilidade de revisão e à participação da sociedade. Conclui-se que o Foundation Document diminui a burocracia e os custos de elaboração de Planos de Manejo e pode ser replicado em outras Unidades de Conservação.

Palavras-chave: Governança; Parque Nacional de São Joaquim; Sociologia pragmática.

Abstract. Foundation Document: A solution in the elaboration of management plans of protected natural areas. This article is a reflection on the elaboration of management plans in protected natural areas in Brazil. We evaluated the adaptation of the

Recebido

$17 / 03 / 2020$

Aceito

28/04/2020

Publicado

$30 / 04 / 2020$

Acesso aberto

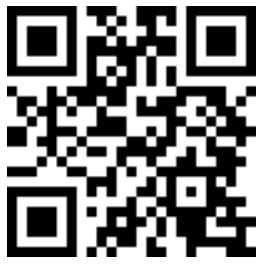

ORCID

(1) 0000-0001-9475-2294 Michel Tadeu R. N. de Omena

ISSN 2359-1412/RBGAS-2020-0042/2020/7/15/22/299

Rev. Bras. Gest. Amb. Sustent.

http://revista.ecogestaobrasil.net 
Foundation Document Model, method used by the US National Park Service in the elaboration of planning documents. This experience was tested by the federal management organ of conservation units in two areas in Brazil, the São Joaquim National Park (Santa Catarina State) and the Soure Marine Extractive Reserve (Pará State). The participant observation method was used in the São Joaquim National Park during the years 2016 to 2018, when its management plan was drawn up, is described here. The use of the adapted Foundation Document Method has shown itself to be more positive than the traditional method in terms of completion time, size, shelf life, ease of review and participation of society. We conclude that foundation document decreased the bureaucracy and costs on the elaboration of Management Plans and it can be replicated in other parks or protected natural areas.

Keywords: Governance; São Joaquim National Park; Pragmatic sociology.

\section{Introdução}

Áreas naturais protegidas são espaços geográficos claramente delimitados, manejados por meio de meios eficazes - jurídicos ou de outra natureza - para alcançar a conservação da natureza em longo prazo, com serviços ecossistêmicos e valores culturais associados (Borrini-Feyerabend et al., 2014); além disso, são uma importante estratégia de conservação da natureza (Magro et al., 2007; Hoffmann et al., 2010; Le Saout et al., 2013; Gray et al., 2016). Porém, apenas a criação de áreas naturais protegidas não garante a proteção. A elaboração de um plano de manejo definindo as diretrizes de gestão deve garantir melhores práticas para a gestão e, consequentemente, poderá refletir positivamente na conservação desses espaços; afinal, há evidências de que áreas naturais protegidas com c são mais conservadas (Chung et al., 2018; Leung et al., 2018).

Infelizmente, por razões diversas, é comum que as áreas naturais protegidas não possuam documentos de planejamento, o que acaba fragilizando a sua gestão. No Brasil, os planos de manejo são o principal instrumento de gestão das áreas naturais protegidas (Brasil, 2000). A construção destes documentos tem sido motivo de grandes debates dentro dos órgãos ambientais, principalmente pela necessidade do aporte de muitas horas de trabalho dos servidores encarregados de sua elaboração (Magro et al., 2007).

Esse é um dos grandes desafios para a gestão das áreas naturais protegidas no Brasil, que abrigam mais de $10 \%$ de toda a biodiversidade mundial (Joly et al., 2019). Apesar de nosso país ser considerado como o primeiro na lista dos países megadiversos (Joly, 2008) e ter $18,1 \%$ de sua porção continental e $26,4 \%$ de sua área marinha protegidos pela legislação das áreas naturais protegidas (Brasil, 2019), enfrenta dilemas e conflitos de interesses em torno dessas áreas. Por isso, considerando a sua expressividade, a governança das áreas naturais protegidas situadas no território brasileiro assume grande relevância também para o processo de conservação da biodiversidade mundial.

A Lei no 9.985/2000 estabeleceu o Sistema Nacional de Unidades de Conservação da Natureza (SNUC) (Brasil, 2000), onde constam as definições legais e categorias para as áreas naturais protegidas, e instituiu o termo unidades de conservação para diferenciar 
estas áreas de terras indígenas ou quilombolas. A lei destaca o conceito oficial para "Plano de Manejo":

[...] documento técnico mediante o qual, com fundamento nos objetivos gerais de uma unidade de conservação, se estabelece o seu zoneamento e as normas que devem presidir o uso da área e o manejo dos recursos naturais, inclusive a implantação das estruturas físicas necessárias à gestão da unidade. (Brasil 2000).

Na prática, porém, os entraves à construção de um plano de manejo são refletidos no número de unidades de conservação com plano de manejo já elaborado. Das 334 unidades de conservação federais brasileiras, 139 (aproximadamente 42\%) não tinham plano de manejo publicado até janeiro de 2019, conforme Instituto Chico Mendes de Conservação da Biodiversidade (ICMBio, 2017). Este baixo número pode ser reflexo da falta de pessoal, o que reflete em tempo excessivo para elaboração e finalização. Como exemplo, podemos citar o caso do Plano de Manejo da Área de Proteção Ambiental da Baleia Franca, cujos conselheiros iniciaram esforços para sua elaboração em 2006, entretanto foi concluído apenas em 2018 (ICMBio, 2018b).

Especificamente, a não elaboração deste documento técnico para as unidades de conservação da categoria parques nacionais, além de prejudicar o planejamento, limita o funcionamento cotidiano e os objetivos destas, pois não permite que sejam realizadas atividades como a visitação, visto que a mesma Lei no 9.985/2000, em seu § 2o, art. 11, prevê que: "[...] a visitação ocorrerá mediante as normas e restrições estabelecidas no seu Plano de Manejo entre outros documentos" (Brasil, 2000).

Tais limitações podem gerar conflitos para a administração dos parques nacionais. Por exemplo, em regiões onde os atrativos dos parques nacionais já fazem parte da cesta de produtos do turismo local (Machado e Costa, 2018), a proibição ao turismo e à visitação por falta de plano de manejo pode impactar diretamente na economia local que depende deste turismo. O Parque Nacional de São Joaquim (PNSJ) em Santa Catarina, criado em 1961 (Brasil, 1961), entre 2012 e 2017 esteve na lista dos dez parques nacionais mais visitados do país; porém, seu plano de manejo foi publicado somente em 2018 (ICMBio, 2018b).

Visando a melhorar essa situação, durante os anos de 2015 e 2016, servidores do ICMBio, do National Park Service (NPS), instituição que administra os parques nacionais estadunidenses e do United States Forest Service (USFS - Serviço Florestal dos Estados Unidos), começaram um intercâmbio de informações que resultou na capacitação de servidores do ICMBio com o objetivo de aplicar nas unidades de conservação brasileiras o modelo utilizado naquele país, o Foundation Document (NPS, 2019). 0 documento alicerce ou de fundação (Foundation Document) tem por objetivo responder questões básicas da gestão das unidades de conservação, tais como qual o propósito do parque? Por que está incluído no sistema nacional? O que isso significa? Quais os recursos e valores fundamentais presentes na área? Quais aspectos ou documentos legais que envolvem a unidade de conservação? Quais são as questões-chave no planejamento e quais dados são necessários? (NPS, 2019).

As duas primeiras áreas a terem seus planos de manejo elaborados a partir deste novo método foram o Parque Nacional de São Joaquim e a Reserva Extrativista Marinha de Soure, no Pará (ICMBio, 2018a; 2018b). Porém, um detalhe importante é que a Lei do SNUC prevê que os planos de manejo devem apresentar um zoneamento das unidades (Brasil, 2000), atividade que não é prevista no Foundation Document. Para cumprir esta e outras exigências legais, foram necessárias adaptações do documento americano para o modelo brasileiro (ICMBio, 2018c).

Neste artigo, relatamos a experiência da adaptação do modelo estadunidense de construção de plano de manejo, o Foundation Document, para a realidade das unidades de 
conservação brasileiras, a partir da vivência prática no processo de construção do plano de manejo do Parque Nacional de São Joaquim. Esta foi uma iniciativa pioneira no país de adaptação do Foundation Document (NPS, 2019), mas um dos desafios para a sua adaptação brasileira foi ampliar a participação da sociedade, concordando com a premissa de que o processo de governança das áreas naturais protegidas somente é legítimo se ocorrer a plena e efetiva participação dos detentores de direitos e dos demais interessados no território, e buscando internalizar um sentimento de pertencimento da área pela sociedade (Borrini-Feyerabend et al., 2014). Esta proposta chama a atenção para a necessidade de uma visão territorialmente abrangente da região ou do país onde ela se situa, enfatizando a importância de uma análise territorial e de sua implantação para o sucesso da governança das áreas naturais protegidas (Raulet-Croset, 2008).

\section{Materiais e métodos}

Nosso método de trabalho teve como base a proposta da Sociologia Pragmática (James et al., 1974; Dewey, 1998) que consiste em observar como os atores produzem suas práticas. Nesta proposta, entender uma prática significa não somente compreender o lugar que ocupam as instituições, os equipamentos técnicos, os processos, os instrumentos, os métodos, mas também uma pluralidade de intencionalidades concorrentes. A prática volta-se, assim, às dimensões social, institucional e técnica nas quais a ação se inscreve (Frega, 2016; Chateauraynaud, 2017).

$\mathrm{Na}$ visão pragmatista, a experiência inclui aquilo que os seres humanos fazem e padecem, aquilo que eles se esforçam para conseguir, mas também como agem e sofrem os efeitos da ação vivenciada (Dewey, 1985; Peirce, 1989). A Sociologia Pragmática busca inspiração para acompanhar as relações sociais através das práticas de ação, almejando compreender como se produzem as intenções, os objetivos, os planos e as perspectivas dos atores envolvidos na ação; em resumo, procura entender como os "atores fazem as coisas" (Chateauraynaud, 2017). Para compreender essa "história das práticas" (Frega, 2016) e nos aproximar do campo, dos atores e da experiência, adotamos como técnica de coleta de dados a observação participante (Bernard, 2006). De uma forma geral, o método de observação participante dá ao pesquisador a oportunidade de se inserir no ambiente da pesquisa e realizar estudos com maior profundidade. Na observação participante, 0 observador se insere na situação social com o objetivo de realizar uma investigação científica, colhendo dados através da relação e sendo parte do contexto, modificando-o e também sendo modificado por ele (Cicourel, 1990).

De acordo com Angrosino e Pérez (2000), as observações realizadas em configurações naturais são as que tendem a causar menor interferência com as pessoas ou atividades sob observação. Assim sendo, duas etapas distintas caracterizaram esse trabalho.

A primeira etapa considerou a participação de dois dos autores deste artigo, também Analistas Ambientais do ICMBio, durante todo o processo de elaboração do plano de manejo, de maio de 2016 a setembro de 2018, com destaque para a oficina de elaboração de Plano de Manejo do Parque Nacional de São Joaquim em agosto de 2016. Além da oficina de elaboração, esses dois Analistas Ambientais participaram da consolidação do documento.

Em uma segunda etapa, entre maio e junho de 2019, foi efetuada uma consulta e análise de questionários online (Apêndice 1) enviados a 23 profissionais-chave do ICMBio, analistas e técnicos ambientais que trabalham diretamente na Coordenação de Elaboração e Revisão de Planos de Manejo (COMAN) ou fazem parte do grupo ampliado de trabalho de elaboração de Planos de Manejo; portanto, com experiência com os roteiros metodológicos de Ibama (2002) ou ICMBio (2011), para elaboração de Planos de Manejo e também com o Foundation Document. A principal pergunta foi: "Comparando o Roteiro Metodológico de 
Planejamento, anteriormente usado, com o Foundation Document, você vê mais vantagens ou desvantagens nesse último e em quais itens?" e foram dadas as opções: mais desvantagens, igual, menos vantagens ou não sei opinar. 0 questionário completo está no Apêndice I. A análise das respostas dadas ao questionário serviram como elemento de reflexão e validação do nosso processo de observação.

Ainda que este documento relate a construção do plano de manejo do Parque Nacional de São Joaquim, foram utilizados como fontes acessórias de informações sobre unidades de conservação com plano de manejo o painel dinâmico de informações do ICMBio (2017).

\section{Área do estudo}

O Parque Nacional de São Joaquim está localizado na região serrana do estado de Santa Catarina, a uma distância de $160 \mathrm{~km}$ da capital, inserindo-se no Bioma Mata Atlântica. 0 parque foi criado em julho de 1961, tendo como principal objetivo a proteção dos remanescentes de Matas de Araucárias, encontradas em abundância dentro de seus 49.800 ha. A área do Parque Nacional abrange os Municípios de Urubici, Bom Jardim da Serra, Orleans, Grão Pará e Lauro Muller.

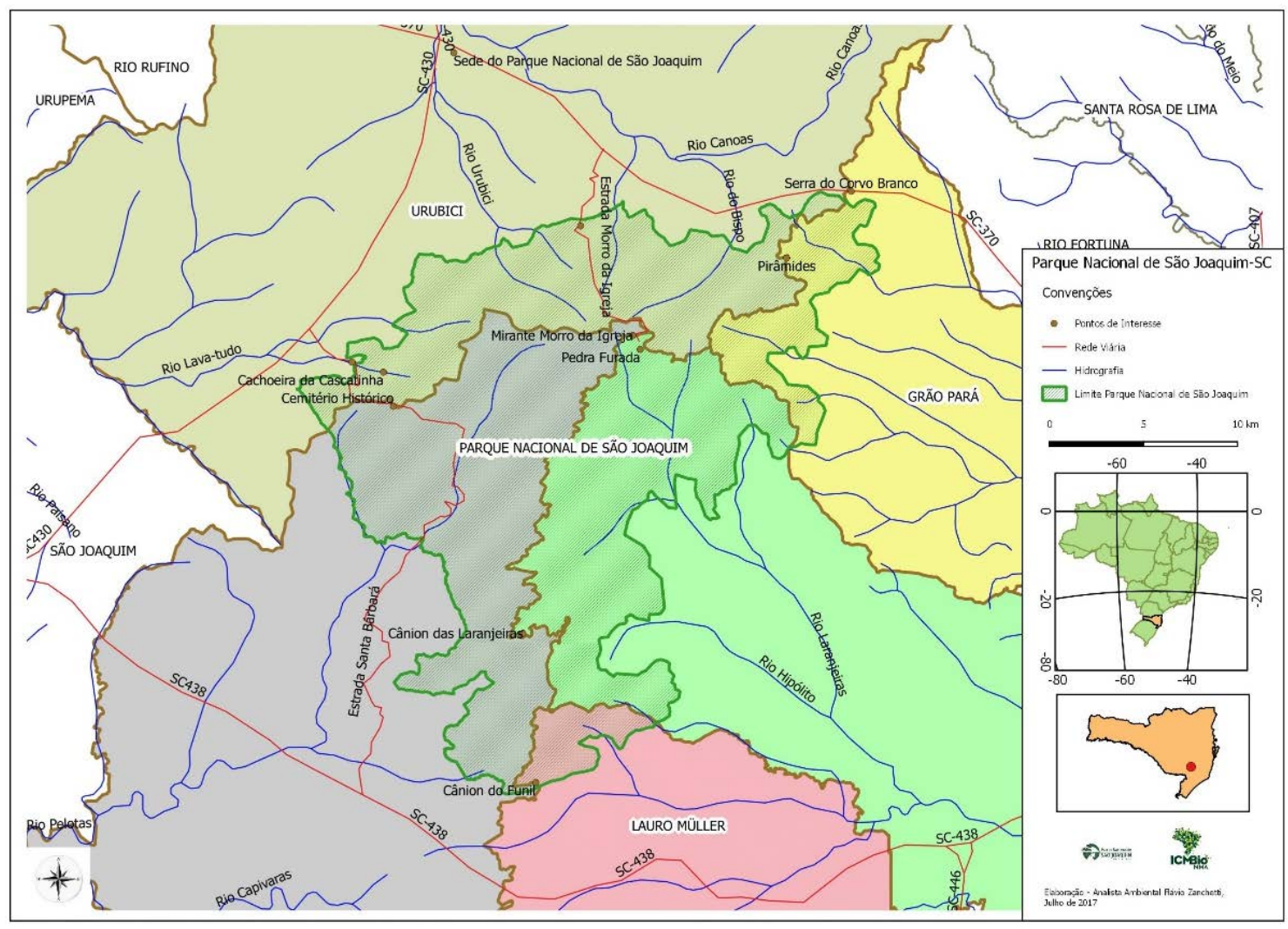

Figura 1. Localização geográfica do Parque Nacional de São Joaquim. (Fonte: ICMBio 2018b).

Atualmente, o Parque Nacional de São Joaquim conta com três servidores públicos Analistas Ambientais, sendo um o chefe da Unidade. Além de funcionários contratados para limpeza, serviços administrativos, vigilância patrimonial e apoio à visitação (12 colaboradores) e temporários (seis meses por ano) para prevenção e combate a incêndios 
florestais (cinco brigadistas). O Parque Nacional de São Joaquim tem conselho consultivo instituído e ativo com 15 membros, há aproximadamente dez anos. Na época da elaboração do plano de manejo, havia apenas dois analistas ambientais ativos.

\section{Resultados e discussão}

Consideramos como início do processo de elaboração do Plano de Manejo a reunião entre os servidores da COMAN, representantes da United States Agency International Development (USAID) e o chefe do Parque Nacional de São Joaquim, ocorrida em Brasília em meados de maio de 2016. A partir de então, foi montada uma equipe técnica com servidores do Parque Nacional de São Joaquim e COMAN que começaram a estruturar a oficina e os documentos de suporte.

Antes da oficina com os atores, foi produzido o "Guia do participante", contendo explicações sobre as etapas do processo de elaboração do Plano de Manejo. Tais etapas compreendiam: como construir uma definição de missão para o Parque Nacional de São Joaquim, definir seus objetivos, recursos e valores fundamentais, questões-chave e necessidades de planejamento. Também foi produzida uma ficha técnica com informações básicas sobre o parque, tais como: tamanho, tipo de vegetação, espécies da fauna e funga, e aspectos geológicos. Assim que foi determinado o período da oficina, foram definidos os convidados pela COMAN e equipe do Parque, buscando-se manter um equilíbrio entre ICMBio, academia, sociedade civil e órgãos públicos. No entanto, na prática, alguns ajustes foram necessários; por exemplo, esperava-se ao menos um representante de órgão público municipal, Prefeitura ou Câmara, de cada uma das cinco cidades que compõem o Parque Nacional de São Joaquim; porém, somente três municípios enviaram representantes. Foram convidados também três conselheiros escolhidos pelo Conselho Consultivo do Parque Nacional de São Joaquim, como se observa na Tabela 1.

Tabela 1. Participantes da oficina de elaboração do Plano de Manejo do Parque Nacional de São Joaquim. (Fonte: Adaptada de ICMBio 2018b).

\begin{tabular}{l|c|l|l}
\hline Representação & Quantos & Observação \\
\hline Participantes da oficina (total 22) & & $\begin{array}{l}\text { Um dos participantes foi indicado pelo Conselho } \\
\text { Consultivo }\end{array}$ \\
\hline Guias/condutores do PNSJ & 3 & Urubici, Bom Jardim da Serra e Grão Pará \\
\hline Prefeituras & 3 & $\begin{array}{l}\text { Um dos participantes foi indicado pelo Conselho } \\
\text { Consultivo }\end{array}$ \\
\hline $\begin{array}{l}\text { Proprietários de áreas } \\
\text { dentro do PNSJ }\end{array}$ & 2 & $\begin{array}{l}\text { Destacamento de Controle do Espaço Aéreo do Morro da } \\
\text { Igreja }\end{array}$ \\
\hline $\begin{array}{l}\text { Força Aérea Brasileira } \\
\text { Academia (Universidades) }\end{array}$ & 3 & $\begin{array}{l}\text { Um dos participantes foi indicado pelo Conselho } \\
\text { Consultivo }\end{array}$ \\
\hline $\begin{array}{l}\text { Órgão Estadual do Meio } \\
\text { Ambiente }\end{array}$ & 1 & $\begin{array}{l}\text { Instituto do Meio Ambiente de Santa Catarina (antiga } \\
\text { FATMA) }\end{array}$ \\
\hline Sociedade Civil & $4(3)$ & $\begin{array}{l}\text { Projeto Papagaio-Charão, } \\
\text { Associação Catarinense de RPPNs, Confederação } \\
\text { Brasileira de Montanhismo e Escalada (CBME) }\end{array}$ \\
\hline $\begin{array}{l}\text { Servidores do ICMBio } \\
\text { Dois do PNSJ, um aposentado e doi de Coordenações } \\
\text { Gerais. }\end{array}$
\end{tabular}


Tabela 1. Continuação.

\begin{tabular}{|c|c|c|}
\hline Representação & Quantos & Observação \\
\hline \multicolumn{3}{|c|}{ Coordenação e logística (total 25) } \\
\hline $\begin{array}{l}\text { Colaboradores } \\
\text { internacionais }\end{array}$ & 5 & $\begin{array}{l}\text { Quatro do NPS (moderadores e geoprocessamento) e um } \\
\text { USFS/USAID (logística) }\end{array}$ \\
\hline Equipe Técnica ICMBio & 5 & $\begin{array}{l}\text { Dois eram os mesmos servidores do PNSJ que atuaram } \\
\text { como participantes da oficina e três da COMAN }\end{array}$ \\
\hline Colaboradores ICMBio & 7 & Equipe de apoio \\
\hline Outros servidores & 6 & Observadores \\
\hline Parlare & 2 & Tradução \\
\hline
\end{tabular}

\section{Durante a oficina}

A equipe técnica e os colaboradores internacionais alinharam os detalhes das etapas do processo, apresentadas na Tabela 2, e especificidades da oficina, como: horários, locais, infraestrutura e divisão de tarefas.

Tablela 2. Resumo do desenvolvimento do trabalho de elaboração do Plano de Manejo do PNSJ.

\begin{tabular}{|l|l|}
\hline Atividade & Ações \\
\hline $\begin{array}{l}\text { Organização prévia à } \\
\text { oficina (de maio a julho de } \\
\text { 2016) }\end{array}$ & $\begin{array}{l}\text { Definir documentos com informações básicas pertinentes à oficina } \\
\text { participativa } \\
\text { Selecionar/contatar participantes } \\
\text { Decisões administrativas (convite aos facilitadores, hospedagem, local } \\
\text { de reuniões etc.) }\end{array}$ \\
\hline $\begin{array}{l}\text { Oficina inicial (agosto de } \\
\text { 2016) }\end{array}$ & $\begin{array}{l}\text { Apresentação e orientações } \\
\text { Visita técnica } \\
\text { Definição de grupos de trabalho por tema } \\
\text { Discussões e elaboração dos documentos iniciais }\end{array}$ \\
\hline Reunião da equipe técnica & Consolidação dos documentos da oficina \\
\hline Grupos de trabalho & $\begin{array}{l}\text { Devolutiva para os grupos de trabalho validarem ou corrigirem, se } \\
\text { pertinente, a consolidação da equipe técnica }\end{array}$ \\
\hline Reunião da equipe técnica & Nova consolidação a partir da devolutiva dos grupos \\
\hline Reunião ampliada & $\begin{array}{l}\text { Equipe técnica apresenta o documento para as Coordenações no } \\
\text { ICMBio sede }\end{array}$ \\
\hline Reunião da equipe técnica & Versão para avaliação jurídica \\
\hline Reunião da equipe técnica & Se necessária, para alterações sugeridas pela Procuradoria Jurídica \\
\hline Aprovação & Deferimento da Diretoria/Presidência do órgão; publicação \\
\hline
\end{tabular}

A experiência prática da atividade foi realizada no município de Urubici. No primeiro dia de trabalho, visando ao reconhecimento do território (Raulet-Croset, 2008), foi feita uma visita técnica com todos os participantes e a coordenação no Parque Nacional de São Joaquim nos municípios de Urubici e Bom Jardim da Serra, com destaque para: a 
estrada e o mirante natural do Morro da Igreja, a Cascata Véu de Noiva, a estrada e alojamento da Santa Bárbara, o Morro do Baú e o Cemitério Tropeiro.

No traslado, com a colaboração de alguns participantes guias de turismo, os servidores do Parque Nacional de São Joaquim fizeram explanações referentes ao Parque, tais como: dados históricos, atividades da Força Aérea no local, fauna e flora, aspectos turísticos e geologia. Essa atividade foi importante para nivelar o conhecimento dos participantes, como também integrar o grupo.

A partir do segundo dia, as atividades se concentraram nas salas disponíveis pela organização, destacando-se uma sala maior para reunião geral dos participantes plenária, onde foi feita a entrega do material de estudo e a apresentações gerais. Neste dia, agregaram-se outros servidores do ICMBio; todavia, na condição exclusiva de observadores, sem, portanto, interferir de qualquer forma nos debates.

0 evento foi dividido em duas partes: a primeira, na qual os moderadores internacionais discutiram os componentes fundamentais do Foundation Document: da definição do propósito, das declarações de significância e dos recursos e valores fundamentais; e a segunda parte, quando foram discutidos os componentes dinâmicos, $o$ zoneamento e as normas (gerais e por zonas), coordenada pela COMAN.

Sempre no início de cada dia, os participantes eram esclarecidos sobre os objetivos do período/dia e divididos em quatro grupos menores.

A cada grupo foi designado um relator. A dinâmica constituía em elaborar o seu entendimento sobre o propósito do Parque Nacional de São Joaquim ou escrever as declarações de significância, que são as características mais importantes da unidade de conservação. Cada grupo também era acompanhado por um facilitador, membro da coordenação, que não podia interferir nas decisões do grupo. No horário marcado, os grupos retornavam a plenária e conciliavam um texto final. Os textos produzidos - por exemplo, sobre o propósito do Parque Nacional de São Joaquim - ficavam em exposição, caso, no transcorrer da oficina, alguém achasse que deveria ser modificado, ou mesmo para ratificar decisões durante a oficina.

Os grupos de atores também entraram em consenso sobre as sete declarações de significância, que justificam os motivos da criação da Unidade e representam os seus recursos e valores fundamentais, como geologia, recursos hídricos, biodiversidade, pesquisa e educação, cultura, turismo e lazer e belezas cênicas (ICMBio, 2018b).

Na segunda parte da oficina, foram tratados os componentes dinâmicos, isto é, aqueles que estão susceptíveis de serem modificados com o passar do tempo. Essa etapa foi coordenada principalmente pelos servidores do ICMBio. Foram elaborados cinco subsídios para a interpretação ambiental: Liberdade de estar em conexão com a natureza, Cultura, Biodiversidade, Beleza Cênica e Cidadania. Os atos e instrumentos legais - tais como: lei do SNUC (Brasil, 2000), lei de criação do Parque Nacional de São Joaquim (Brasil, 1961) e lei de alteração dos limites do Parque Nacional de São Joaquim (Brasil, 2016) foram repassados à plenária para registro, mas também como subsídio para as etapas posteriores.

Com os valores e recursos fundamentais conhecidos e pactuados entre os presentes, foi necessária a avaliação das necessidades de dados e planejamento e oportunidades e ações de manejo, para cada um dos sete valores e recursos. Coincidentemente é o mesmo número de declarações de significância (sete). Neste momento da oficina, os grupos foram reorganizados de acordo com o interesse de cada participante, em grupos com número de membros aproximadamente igual.

Cada grupo ficou incumbido de levantar a situação atual, as tendências, as ameaças e o nível de informações disponível sobre o recurso ou valor. Por exemplo, o grupo que trabalhou o tema Cultura, identificou falta de sinalização e uso desordenado de áreas historicamente relevantes, a tendência era a visitação danificar o patrimônio cultural e isso se tornar uma ameaça cada vez maior, pelo aumento do número de visitantes. 0 grupo 
identificou um material sobre cavernas no Parque Nacional de São Joaquim, mas os sítios arqueológicos não se encontravam catalogados. A partir desta discussão, o grupo definiu a necessidade de dados e de planejamento, e dentro destes itens as prioridades foram: baixa, média ou alta. Na sequência foram elencadas as oportunidades de ações e manejo, também as classificando em prioridades.

Esse procedimento foi feito para cada um dos sete recursos e valores fundamentais: Geologia, Recursos Hídricos, Biodiversidade, Pesquisa e Educação, Cultura, Turismo e Lazer, e, por fim, Belezas Cênicas. Posteriormente cada grupo expôs as suas conclusões na plenária, sendo construído um texto final.

Na sequência, foram levantadas as questões-chave que comprometem a gestão do Parque Nacional de São Joaquim. Semelhante ao que foi feito anteriormente, as seguintes questões foram avaliadas em grupos menores, Regularização Fundiária, Parques "Fortaleza" (termo usado para definir parques fechados à visitação), Limite do Parque (redefinição e incerteza), Comunicação com o entorno e Acesso de pessoas não autorizadas. Para todas as questões, foi levantada a situação atual; depois, foram levantadas as necessidades de dados e planejamento, assim como as oportunidades de ações e manejo.

A parte final da elaboração do Plano de Manejo foi a construção do zoneamento e suas regras. Esta etapa foi precedida por uma explanação sobre o conceito de zoneamento e suas categorias. Novamente os participantes foram divididos em grupos menores, que tiveram o apoio de especialistas em geoprocessamento para produzirem um mapa de zoneamento de acordo com o conhecimento de cada um sobre a região do Parque Nacional de São Joaquim. As quatro propostas de zoneamento foram apresentadas à plenária, que não validou integralmente nenhuma delas. As críticas e sugestões foram anotadas, e a equipe técnica ficou responsável por formatar uma proposta de consenso, concluída após ser aprovada posteriormente pelos participantes.

Tendo fixado o conceito de zoneamento, a partir de exemplos fornecidos pela coordenação, os grupos se reuniram novamente e criaram estratégias de debate diferentes para a elaboração das regras das zonas. Dois dos quatro grupos se prenderam aos materiais entregues pela organização no início da oficina e fizeram uma normatização geral e outra por zona; outro grupo pensou apenas no geral; e o quarto grupo criou normas independentes do material apresentado. Após a compilação do material, em plenária estas normas foram ratificadas ou excluídas.

Ao final de cada dia de oficina, o grupo de moderadores e observadores se reunia para discutir os avanços e contratempos do dia, tomando medidas corretivas de imediato. Todo o material produzido, como cartazes, fotos e mapas, foi arquivado no Parque Nacional de São Joaquim para consultas posteriores.

Nesta etapa presencial ficou evidente o comprometimento dos participantes que não faltaram em nenhum dos dias e chegavam antecipadamente ao horário de início $(8 \mathrm{~h})$ e permaneciam até o seu final $(17 \mathrm{~h})$, aparentemente com o mesmo entusiasmo. Esse comprometimento indica que a dinâmica da oficina não só atingiu seu objetivo principal de elaboração de um documento preliminar do Plano de Manejo mas também se mostrou eficiente para que os moderadores conseguissem engajamento dos participantes.

Quando da elaboração do Foudation Document pelo NPS para as unidades de conservação estadunidenses, todo o trabalho é exclusivo de técnicos e consultores, deixando para uma etapa posterior do processo a participação da sociedade. Assim, no trabalho de adaptação do modelo estadunidense para a realidade brasileira, tivemos de trazer para o processo a premissa da participação da sociedade desde seu início, que é também uma das diretrizes do ICMBio para a elaboração dos planos de manejo, objetivando maior participação da sociedade no processo de governança das unidades de conservação. 


\section{Após a oficina}

Para cada valor ou recurso fundamental proposto na oficina, a equipe técnica formatou quadros de priorização e listou: condições atuais, tendências, ameaças e status das informações. Logo após, para cada um dos recursos e valores fundamentais, foram levantadas:

a) Necessidades de dados e/ou sistema de informação geográfica (SIG) e prioridade inicial;

b) Necessidades de planejamento e prioridade inicial; e

c) Oportunidades e ações de manejo, incluindo prioridade inicial, anotando para cada ação o grau de prioridade (baixa, média e alta) - exemplo pode ser visto na Tabela 3.

Tabela 3. Parte do quadro de recursos e valores fundamentais, item "Belezas Cênicas", como exemplo do Plano de Manejo do Parque Nacional de São Joaquim (Fonte: ICMBio 2018b).

\begin{tabular}{|c|c|}
\hline \multicolumn{2}{|l|}{ Belezas Cênicas } \\
\hline \multicolumn{2}{|c|}{$\begin{array}{l}\text { Condições atuais } \\
\text { - Parque sofre pressão da sociedade para aumento da visitação } \\
\text { - Degradação de turfeiras, altamente impactadas pela visitação excessiva, como ocorre no } \\
\text { morro da Igreja, e pelo excesso de veículos } 4 x 4 \text {, como observado no cânion do Funil e nos } \\
\text { Campos de Santa Bárbara (jipeiros) }\end{array}$} \\
\hline \multicolumn{2}{|c|}{$\begin{array}{l}\text { Tendências } \\
\text { - Continuarão ocorrendo alterações pela visitação excessiva e uso desordenado por veículos } \\
\text { (4x } 4 \text { e motocicletas), como abertura de novos caminhos e degradação de turfeiras e } \\
\text { outros solos em geral, conforme se observa no morro da Igreja, nos Campos de Santa } \\
\text { Bárbara e no cânion do Funil } \\
\text { - Aumento da pressão da sociedade para alteração dos limites do Parque, como é o caso das } \\
\text { áreas do cânion do Funil e da cascata Véu de Noiva }\end{array}$} \\
\hline \multicolumn{2}{|c|}{$\begin{array}{l}\text { Ameaças } \\
\text { - Aumento da visitação e pressão da sociedade para maior uso } \\
\text { - Aumento dos resíduos sólidos (lixo) e degradação dos solos pelo pisoteio, no morro da } \\
\text { Igreja, na Travessia e nos Campos de Santa Bárbara }\end{array}$} \\
\hline \multicolumn{2}{|c|}{$\begin{array}{l}\text { Status de informação } \\
\text { • A informação existente é insuficiente, desatualizada e encontra-se fragmentada }\end{array}$} \\
\hline $\begin{array}{l}\text { Necessidades de dados e/ou sistema de Informação geográfica (SIG) e } \\
\text { prioridade inicial (alta, média ou baixa) }\end{array}$ & Prioridade \\
\hline - Atualização do inventário e mapeamento dos atrativos e trilhas (SIG) & Alta \\
\hline - Monitoramento do impacto da visitação & Média \\
\hline \multicolumn{2}{|l|}{ Necessidades de planejamento e prioridade inicial } \\
\hline - Conclusão da regularização fundiária de áreas vulneráveis/atrativos & Alta \\
\hline - $\quad$ Plano de recuperação de áreas degradadas & Baixa \\
\hline \multicolumn{2}{|l|}{ Oportunidades e ações de manejo, incluindo prioridade inicial } \\
\hline - $\quad$ Capacitar condutores de visitantes e multiplicadores & Alta \\
\hline $\begin{array}{l}\text { - Conscientizar os visitantes quanto à importância do parque e de seus } \\
\text { recursos (folheto, placa, saco de lixo etc.) }\end{array}$ & Média \\
\hline
\end{tabular}


Os quadros de priorização, zoneamento e normas foram reapresentados aos participantes da oficina para uma segunda rodada de discussões após cerca de 90 dias, quando se manifestaram deferindo ou não da primeira versão do texto.

A devolução das observações da segunda rodada pelos participantes da oficina ocorreu cerca de 60 dias após o recebimento da primeira versão. Passado este prazo, a equipe técnica compilou o material e apresentou a versão final. Dos 62 itens levantados, 43,54\% foram listados como prioridade alta; 37,10\%, média; e 19,35\%, baixa (Figura 2). Estes dados apontam que houve um processo criterioso dentro dos grupos, para não colocarem todos os itens como prioridade alta.

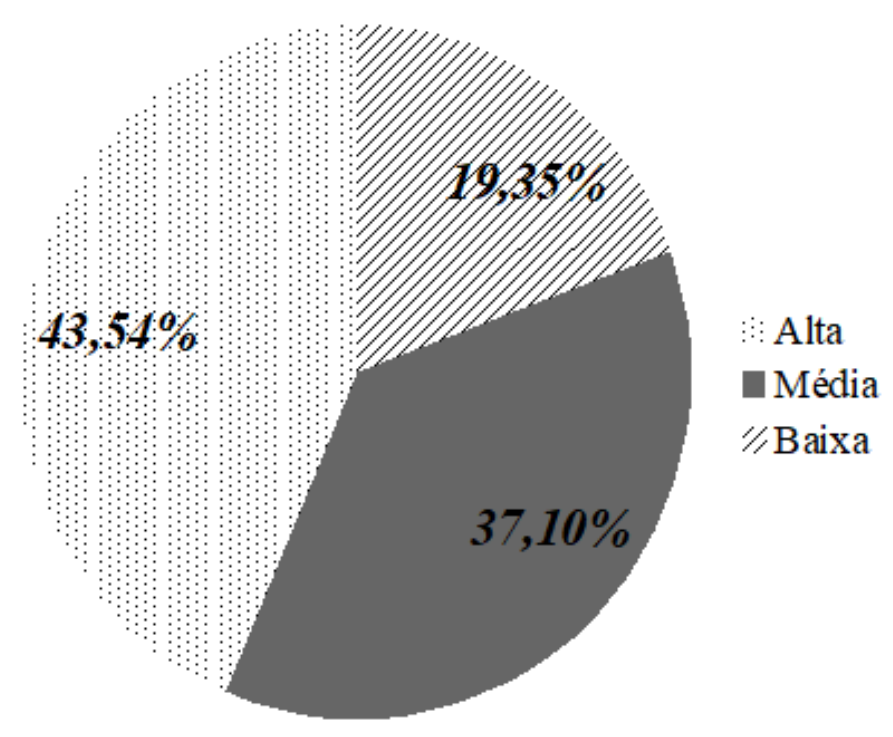

Figura 2. Porcentagem de itens definidos por níveis de prioridade para o Plano de Manejo do Parque Nacional de São Joaquim, Brasil.

Após reuniões da equipe técnica, em julho de 2017 ocorreu uma reunião presencial com os diretores e coordenadores do ICMBio. Nesta etapa, uma importante resolução foi sustentar as decisões tomadas pelos participantes, e assim os ajustes feitos não representaram situações que comprometessem a participação da sociedade.

Após análise jurídica, feita pela Procuradoria Especializada do ICMBio, o documento sofreu uma alteração importante que foi a retirada das normas da zona de amortecimento, assim como sua definição, ainda que no formato de proposta.

0 documento final foi publicado em 21 de setembro de 2018 e está disponível noa página oficial do ICMBio na Internet. Desde o seu início, em maio de 2016, até a conclusão do texto em agosto de 2017, a sua elaboração demorou apenas um ano e meio, tornandose o primeiro Plano de Manejo de Parque Nacional que utilizou a abordagem baseada no Foundation Document.

\section{Consulta com questionários}

As perguntas comparativas entre os Roteiros Metodológicos do ICMBio utilizados anteriormente para elaboração de planos de manejo - referências como Ibama (2002), ICMBio (2011) - e o Foundation Document foram enviadas a 23 profissionais-chave, dos quais 14 retornaram o questionário preenchido.

Na percepção dos profissionais consultados, o Foundation Document apresenta mais vantagens quanto ao número de servidores envolvidos, custo e tempo total de elaboração, à facilidade de revisão e ao atendimento aos objetivos. Quanto à participação 
social, é semelhante ao modelo tradicional; mas, quanto à necessidade de produzir outros documentos no futuro, foi considerado desvantajoso (Figura 3).

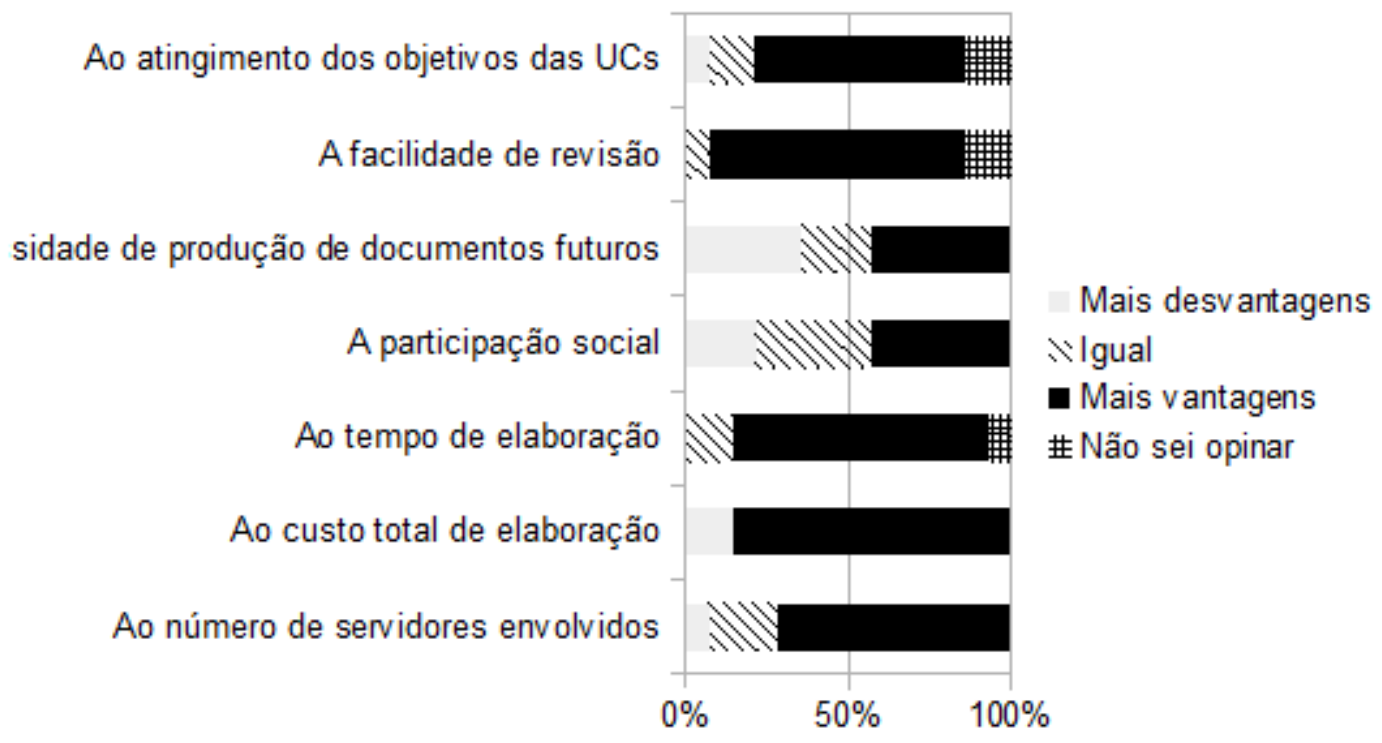

Figura 4. Comparação entre o roteiro metodológico para elaboração de planos de manejo, anteriormente usado pelo ICMBio, com o Foundation Document (percepção de 14 entrevistados).

Há que se destacar que os profissionais citaram, por diversas vezes, como alternativa metodológica ao Roteiro Metodológico de Elaboração de plano de manejo, os "Padrões Abertos para a Prática da Conservação", desenvolvido pela Aliança para as Medidas de Conservação, segundo Conservation Measures Partnership (CMP, 2007). Muito resumidamente, esse método pode ser dividido em cinco atividades: 1) descrição do que se pretende alcançar, 2) identificação de quais ações são necessárias e como monitorá-las, 3) implementação de ações e monitoramento, 4) análise dos dados e correções necessárias, e 5) documentação e divulgação de resultados para disseminar o aprendizado.

Os resultados dos questionários também apontam para mais vantagens com uso do Foundation Document, (Tabela 4).

Do ponto de vista legal, o Parque Nacional de São Joaquim já pode ter visitação, pois o seu Plano de Manejo foi publicado. Este documento autoriza, mesmo que de forma geral, a visitação na área, aguardando a produção do Plano de Uso Público para o detalhamento das estratégias para visitação.

A falta de documentos de planejamento e monitoramento estão entre as maiores deficiências das áreas naturais protegidas, de acordo com um levantamento feito em mais de 4.000 delas (Leverington et al., 2010), e consequentemente comprometem o atingimento de seus objetivos (Instituto Semeia, 2019). No Brasil, a lei do SNUC (Brasil, 2000), que regulamenta as áreas naturais protegidas, prevê que estas tenham um Plano de Manejo, onde deve constar o zoneamento das Unidades, atividade que não é prevista no Foundation Document (NPS, 2019). Para cumprir esta e outras exigências legais, foram necessárias adaptações do documento americano para o modelo brasileiro, ressaltando-se que, além de instrumento de gestão, legalmente previsto, a elaboração do plano de manejo é também um dos 22 objetivos estratégicos do ICMBio (2019). 
Tabela 4. Comparação entre Planos de Manejo construídos no modelo tradicional e pela abordagem baseada no Foundation Document.

\begin{tabular}{|l|l|l|}
\hline Item & $\begin{array}{c}\text { Roteiro Metodológico anterior } \\
\text { (Ibama, 2002; ICMBio, 2011) }\end{array}$ & $\begin{array}{c}\text { Foundation Document (NPS, } \\
\text { 2019) }\end{array}$ \\
\hline Tempo de produção & Normalmente maior que dois anos & Cerca de um ano e meio, \\
\hline Necessidade de revisão & A cada cinco ou dez anos & Sem limite \\
\hline Participação da sociedade & Depende do método & Alto índice de participação \\
\hline Tamanho do documento & Grande, centenas de páginas & Pequeno, cerca de 55 páginas \\
\hline Detalhamento & Bastante detalhado & Resumido \\
\hline Interpretação & Complexo & Fácil leitura \\
\hline $\begin{array}{l}\text { Necessidade de documentos } \\
\text { extras }\end{array}$ & Pouca & Média \\
\hline Atende aspectos legais & Sim & \\
\hline Necessidade de servidores & Semelhante & \\
\hline
\end{tabular}

Embora seja uma determinação legal, a elaboração destes documentos sempre foi problemática, mesmo quando as unidades de conservação eram administradas pelo Instituto Brasileiro do Meio Ambiente e dos Recursos Naturais Renováveis (Ibama), e agora também pelo ICMBio (Brasil, 2007). Na prática, os órgãos ambientais não têm conseguido atender essa exigência, pois isso necessita do envolvimento direto dos seus servidores, que estão comprometidos normalmente com diversas tarefas cotidianas. Um quinto dos Parques Nacionais tem dois ou menos servidores (Instituto Semeia, 2019). Outro problema é a complexidade, que envolve a participação de diversos atores da sociedade e, consequentemente, diferentes interesses.

0 conflito de interesses entre os diversos atores sociais sobre os objetivos que devem ter as unidades de conservação pode ser considerado um problema público (Dewey, 1998) e também uma questão de gestão territorial (Raulet-Croset, 2008). Nesta arena, além dos interesses voltados para as questões ambientais, encontram-se atores que representam as apostas de diversos segmentos e instituições, o que muitas vezes gera conflitos que não têm como objetivo a conservação da natureza e dos ecossistemas.

Assim, mais relevante se torna a participação popular, mesmo quando os propósitos de alguns atores possam ser incompatíveis com os objetivos de conservação da biodiversidade - por exemplo, quando há interesses de mineração, uma atividade que gera impactos ambientais diretos. Porém, há alternativas bem sucedidas de participação social mesmo quando existem interesses conflitantes. Convém mencionar que, na Finlândia, um sistema de gerenciamento social para áreas com mineração tem se mostrado valioso em administrar todos os conflitos de interesse (Suopajärvia e Kantola, 2020).

Neste processo, duas questões (Tabela 2) dificultam o engajamento de parte da comunidade nas ações de proteção do território (Raulet-Croset, 2008). Uma delas é a "regularização fundiária", que, no caso do Parque Nacional de São Joaquim, ganhou destaque e teve várias de suas demandas enquadradas como prioridade "alta". E este assunto é considerado um entrave à gestão das unidades de conservação (Magro et al., 2007; Instituto Semeia, 2019). A outra questão é o processo de "definição de limites", que no Parque Nacional de São Joaquim aparece como uma questão-chave isolada.

Neste ponto, destaca-se que a coordenação feita pelos moderadores internacionais foi essencial para a neutralidade do processo de construção do Plano de Manejo. 0 processo de internalização das unidades de conservação junto à sociedade é representado 
pela democratização e a descentralização das decisões (Bezerra et al., 2018). A atuação dos moderadores internacionais evitou que preconceitos arraigados em parte do grupo interferissem no processo. 0 processo participativo ocorrido na oficina foi muito importante, porque nem sempre os governos são abertos ao diálogo, como ocorre na China, onde as decisões são top-down e se exclui a sociedade das discussões e, consequentemente, do processo de elaboração de planos de manejo para seus parques nacionais (Wang, 2019).

Ainda que tenha sido fundamental a participação de profissionais estadunidenses, o que mais se destaca no novo método é a redução do tempo de elaboração do documento. Alguns dos aspectos mais frágeis da gestão de áreas naturais protegidas são o seu número de funcionários e a adequação de recursos financeiros (Leverington et al., 2010). Dessa forma, um documento mais conciso diminui o tempo de elaboração e também, posteriormente, o de consulta, tornando-se mais acessível para a sociedade e novos colaboradores. Sua revisão consequentemente pode ser mais rápida, principalmente se considerarmos necessidades que podem surgir a partir das perspectivas de mudanças ambientais rápidas, por exemplo, aquelas decorrentes das variações climáticas.

A comunidade científica alerta sobre as alterações que as mudanças climáticas vão ocasionar aos ecossistemas, como mudanças na vegetação e recursos hídricos (Peñuelas et al., 2018; Rogora et al., 2018), e isso reflete no manejo das áreas naturais protegidas. Políticas públicas prevendo alterações provocadas pelas mudanças climáticas já estão sendo pensadas no mundo (O'Donnell, 2019) e os modelos anteriores de elaboração de planos de manejo (Ibama, 2002; ICMBio, 2011), devido à alta complexidade e dificuldade de revisão, também comprometem os objetivos de conservação frente a essas mudanças.

Um documento mais conciso como o Foundation Document mostra-se muito útil à gestão das unidades de conservação. Entretanto, especificamente no caso do Parque Nacional de São Joaquim - onde as diretrizes sobre a zona de amortecimento foram excluídas do processo - fragilizou-se a proteção da Unidade. Por conseguinte, instituir uma zona de amortecimento é uma importante estratégia de mitigação de impactos as áreas naturais protegidas (Lima e Ranieri, 2018).

\section{Conclusões}

Processos burocráticos estão quase sempre atrelados à má gestão. E, no caso das áreas naturais protegidas, eles são prejudiciais aos objetivos de conservação. De acordo com as nossas percepções, ratificadas pelas entrevistas realizadas, a elaboração de planos de manejo pela adaptação do Foundation Document traz vantagens em relação aos modelos anteriores. Os pontos positivos a serem destacados são: maior participação da sociedade, redução do tempo de elaboração, concentração de esforços nos principais recursos e valores, priorização de ações e manejo; o que resulta na produção de um documento mais conciso e, portanto, acessível a mais pessoas. Por outro lado, o novo modelo deixa a elaboração de alguns documentos - como planos de uso público e gerenciamento do fogo - para uma etapa posterior; mas, efetivamente, eles podem ser produzidos com um esforço menor e de acordo com as prioridades que o Plano de Manejo definiu. Concluímos que o método pode e deve ser replicado em outras situações, pois essa nova proposta de construção de Planos de Manejo se mostrou eficiente e apresentou benefícios quando comparada com modelos anteriormente usados.

\section{Agradecimentos}

Agradecemos a todos os participantes da oficina de elaboração do Plano de Manejo do Parque Nacional de São Joaquim, aos servidores do ICMBio, NPS e USFS, com destaque para os colegas: Lourdes M. Ferreira, Edilene Oliveira de Menezes e Flávio Zanchetti. 


\section{Conflito de interesses}

Os autores declaram não haver conflito de interesses.

\section{Referências}

Angrosino, M. V.; Pérez, K. A. M. Rethinking observation from method to context. In: Denzin, N. K.; Lincoln, Y. S. (Eds.). Handbook of qualitative research. 5. ed. Thousand Oaks: Sage, 2000.

Bernard, H. R. Research methods in Anthropology: Qualitative and quantitative approaches. 4. ed. New York: Altamira Press, 2006.

Bezerra, G. S. C. L.; Carvalho, R. M. C. M. O.; Lyra, M. R. C. C.; Frutuoso, M.N.M.A. \& Rodrigues, S. S. F. B. Política pública, participação social e gestão de unidades de conservação: novos caminhos para antigos desafios. Holos, v. 6, p. 117-129. 2018. https://doi.org/10.15628/holos.2018.4486

Borrini-Feyerabend, G.; Dudley, N.; Jaeger, T.; Lassen, N.; Broome, N. P.; A. Phillips, A.; Sandwith, T. Gobernanza de áreas protegidas: de la comprensión a la acción. Gland, Suiza: IUCN, 2014. Disponível em: <https://www.iucn.org/es/content/gobernanza-deareas-protegidas-de-la-comprension-a-la-accion>. Acesso em: 15 jan. 2019.

Brasil. Decreto no 50.922, de 06 de julho de 1961. Cria o Parque Nacional de São Joaquim no Estado de Santa Catarina, e dá outras providências. Disponível em: <https://www2.camara.leg.br/legin/fed/decret/1960-1969/decreto-50922-6-julho1961-391124-norma-pe.html>. Acesso em: 15 jan. 2019.

Brasil. Lei no 11.516, de 28 de agosto de 2007. Dispõe sobre a criação do Instituto Chico Mendes de Conservação da Biodiversidade - Instituto Chico Mendes; altera as Leis nos 7.735, de 22 de fevereiro de 1989, 11.284, de 2 de março de 2006, 9.985, de 18 de julho de $2000,10.410$, de 11 de janeiro de 2002, 11.156, de 29 de julho de 2005, 11.357, de 19 de outubro de 2006, e 7.957, de 20 de dezembro de 1989; revoga dispositivos da Lei no 8.028, de 12 de abril de 1990, e da Medida Provisória no 2.216-37, de 31 de agosto de 2001; e dá outras providências. Disponível em: <http://www.planalto.gov.br/ccivil_03/_Ato20072010/2007/Lei/L11516.htm>. Acesso em: 15 jan. 2019.

Brasil. Lei no 13.273, de 15 de abril de 2016. Altera os limites do Parque Nacional de São Joaquim, no Estado de Santa Catarina. Disponível em: <http://www.planalto.gov.br/ ccivil_03/_ato2015-2018/2016/lei/L13273.htm>. Acesso em: 15 jan. 2019.

Brasil. Lei no 9.985, de 18 de julho de 2000. Regulamenta o art. 225, § 1ํㅡ, incisos I, II, III e VII da Constituição Federal, institui o Sistema Nacional de Unidades de Conservação da Natureza e dá outras providências. Disponível em: <http://www.planalto.gov.br/ ccivil_03/leis/19985.htm>. Acesso em: 15 jan. 2019.

Brasil. Ministério do Meio Ambiente. 2019. Dados Consolidados. Disponível em: <http://www.mma.gov.br/areas-protegidas/cadastro-nacional-de-ucs/dadosconsolidados.html/>. Acesso em: 10 jul. 2019.

Chateauraynaud, F. A Captura como experiência, investigações pragmáticas e teorias do poder. Revista Brasileira de Ciências Sociais, v. 32, 2017. https://doi.org/10.17666/ $329504 / 2017$

Chung, M.; Dietz, T.; Liu, J. Global relationships between biodiversity and nature-based tourism in protected areas. Ecosystem Services, v. 34, Part A, p. 11-23, 2018. https://doi.org/10.1016/j.ecoser.2018.09.004

Rev. Bras. Gest. Amb. Sustent., 2020, vol. 7, n. 15, p. 299-317. 
Cicourel, A. Teoria e método em pesquisa de campo. In: Guimarães, A. Z. (Org.). Desvendando máscaras sociais. São Paulo: Francisco Alves. 1990. p. 87-121.

CMP - Conservation Measures Partnership. Padrões abertos para a prática da conservação. The Conservation Measures Partnership. USAID. Versão 2.0., 2007.

Dewey, J. Excertos de "O público e seus problemas" (1927). In: Hickmean, L. A.; Alexander, T. The Essential Dewey, vol. 1: Pragmatism, education, democracy. Indiana: Indiana University Press, 1998.

Dewey, J. Experiência e natureza. Lógica: a teoria da investigação. A arte como experiência. Vida e educação. São Paulo: Abril Cultural, 1985.

Frega, R. Qu'est-ce qu'une pratique? In: Cohen, Y.; Chateauraynaud, F. Histoires pragmatiques. Paris: EHESS, 2016.

Gray, C. L.; Hill, S. L. L.; Newbold, T.; Hudson, L. N.; Börger, L.; Contu, S.; Hoskins, A.J.; Ferrier, S.; Purvis, A.; Scharlemann, J. P. W. Local biodiversity is higher inside than outside. Nature Commmunications, v. 7, Article number 12306, 2016. https://doi.org/10.1038/ ncomms 12306

Hoffmann, M.; Hilton-Taylor, C.; Angulo, A.; Böhm, M.; Brooks, T. M.; Butchart, S. H. M.; Carpenter, K. E.; Chanson, J.; Collen, B.; Cox, N. A.; Darwall, W. R. T.; Dulvy, N. K.; Harrison, L. R.; Katariya, V.; Pollock, C. M.; Quader, S.; Richman, N. I.; Rodrigues, A. S. L.; Tognelli, M. F.; Vié, J.-C.; Aguiar, J. M.; Allen, D. J.; Allen, G. R.; Amori, G.; Ananjeva, N. B.; Andreone, F.; Andrew, P.; Ortiz, A. L. A.; Baillie, J. E. M.; Baldi, R.; Bell, B. D.; Biju, S. D.; Bird, J. P.; BlackDecima, P.; Blanc, J. J.; Bolaños, F.; Bolivar-G., W.; Burfield, I. J.; Burton, J. A.; Capper, D. R.; Castro, F.; Catullo, G.; Cavanagh, R. D.; Channing, A.; Chao, N. L.; Chenery, A. M.; Chiozza, F.; Clausnitzer, V.; Collar, N. J.; Collett, L. C.; Collette, B. B.; Fernandez, C. F. C.; Craig, M. T.; Crosby, M. J.; Cumberlidge, N.; Cuttelod, A.; Derocher, A. E.; Diesmos, A. C.; Donaldson, J. S.; Duckworth, J. W.; Dutson, G.; Dutta, S. K.; Emslie, R. H.; Farjon, A.; Fowler, S.; Freyhof, J.; Garshelis, D. L.; Gerlach, J.; Gower, D. J.; Grant, T. D.; Hammerson, G. A.; Harris, R. B.; Heaney, L. R.; Hedges, S. B.; Hero, J.-M.; Hughes, B.; Hussain, S. A.; Icochea M., J.; Inger, R. F.; Ishii, N.; Iskandar, D. T.; Jenkins, R. K. B.; Kaneko, Y.; Kottelat, M.; Kovacs, K. M.; Kuzmin, S. L.; La Marca, E. ; Lamoreux, J. F.; Lau, M. W. N.; Lavilla, E. O.; Leus, K.; Lewison, R. L.; Lichtenstein, G.; Livingstone, S. R.; Lukoschek, V.; Mallon, D. P.; McGowan, P. J. K.; McIvor, A.; Moehlman, P. D.; Molur, P. D.; Alonso, A. M.; Musick, J. A.; Nowell, K.; Nussbaum, R. A.; Olech, W.; Orlov, N. L.; Papenfuss, T. J.; Parra-Olea, G.; Perrin, W. F.; Polidoro, B. A.; Pourkazemi, M.; Racey, P. A.; Ragle, J. S.; Ram, M.; Rathbun, G.; Reynolds, R. P.; Rhodin, A. G. J.; Richards, S. J.; Rodríguez, L. O.; Ron, S. R.; Rondinini, C.; Rylands, A. B.; Sadovy de Mitcheson, Y.; Sanciangco, J. C.; Sanders, K. L.; Santos-Barrera, G.; Schipper, J.; Self-Sullivan, C.; Shi, Y.; Shoemaker, A.; Short, G.; Sillero-Zubiri, C.; Silvano, D. L.; Smith, K. G.; Smith, A. T.; Snoeks, J.; Stattersfield, A. J.; Symes, A. J.; Taber, A. B.; Talukdar, B. K.; Temple, H. J.; Timmins, R.; Tobias, J. A.; Tsytsulina, K.; Tweddle, D.; Ubeda, C.; Valenti, S. V.; van Dijk, P. P.; Veiga, L. M.; Veloso, A.; Wege, D. C.; Wilkinson, M.; Williamson, E. A.; Xie, F.; Young, B. E.; Akçakaya, H. R.; Bennun, L.; Blackburn, T. M.; Boitani, L.; Dublin, H. T.; Fonseca, G. A. B.; Gascon, C.; Lacher Jr., T. E.; Mace, G. M.; Mainka, S. A.; McNeely, J. A.; Mittermeier, R. A.; Reid, G. M.; Rodriguez, J. P.; Rosenberg, A. A.; Samways, M. J.; Smart, J.; Stein, B. A.; Stuart, S. $\mathrm{N}$. The impact of conservation on the status of the world's vertebrates. Science, v. 330, n. 6010, p. 1503-1509, 2010. https://doi.org/10.1126/science.1194442

IBAMA - Instituto Brasileiro do Meio Ambiente e dos Recursos Naturais Renováveis. Roteiro metodológico de planejamento: parques nacionais, reservas biológicas e estações ecológicas. Brasília: IBAMA, 2002. 
ICMBio - Instituto Chico Mendes de Conservação da Biodiversidade. Roteiro metodológico de planejamento: parques nacionais, reservas biológicas e estações ecológicas. Brasília: ICMBio, 2011.

ICMBio - Instituto Chico Mendes de Conservação da Biodiversidade. 2017. Painel Dinâmico de informações. Disponível em: <http://qvicmbio.gov.br/QvAJAXZfc/opendoc2.htm? document=painel_corporativo_6476.qvw\&amp;host=Local\&amp;anonymous=true >.

Acesso em: 15 jan. 2019.

ICMBio - Instituto Chico Mendes de Conservação da Biodiversidade. Portaria no 712, de 13 de agosto de 2018a. Aprova o Plano de Manejo da Reserva Extrativista Marinha de Soure, no Estado do Pará (Processo no 02127.012546/2017-61). Disponível em: <https://www.icmbio.gov.br/portal/images/stories/portaria_712_13ago2018_aprova_pla no_de_manejo_rexes_soure.pdf $>$. Acesso em: 15 jan. 2019.

ICMBio - Instituto Chico Mendes de Conservação da Biodiversidade. Portaria no 811, de 21 de setembro de 2018b. Aprova o Plano de Manejo do Parque Nacional de São Joaquim (Processo no 02127.000110/2011-31). Disponível em: <https://www.icmbio.gov.br/ portal/images/stories/portarias/portaria_811_21_de_setembro_2018.pdf >. Acesso em: 15 jan. 2019.

ICMBio - Instituto Chico Mendes de Conservação da Biodiversidade. Roteiro metodológico para elaboração e revisão de planos de manejo das unidades de conservação federais. Brasília: ICMBio, 2018c.

ICMBio - Instituto Chico Mendes de Conservação da Biodiversidade. Gestão Estratégica. 2019. Disponível em: <http://www.icmbio.gov.br/portal/gestaoestrategica>. Acesso em: 15 ago. 2019.

Instituto Semeia. Diagnóstico do uso público em parques brasileiros: a perspectiva dos gestores. São Paulo: Instituto Semeia, 2019. Disponível em: <http://bit.ly/2nVc0xl>. Acesso em: 15 jan. 2019.

James, W.; Dewey, J.; Veblen, T. Pragmatismo: textos selecionados. São Paulo: Abril Cultural, 1974.

Joly, C. Biodiversity and climate change in the Neotropical region. Biota Neotropica, v. 8, n. 1, p. 1-3, 2008. https://doi.org/10.1590/S1676-06032008000100001

Joly, C.; Scarano, F. R.; Seixas, C. S.; Metzger, J. P.; Ometto J. P.; Bustamante, M. C. M.; Padgurschi, M. C. G.; Pires, A. P. F.; Castro, P. F. D.; Gadda, T.; Toledo, P. (Eds.). Primeiro diagnóstico brasileiro de biodiversidade e serviços ecossistêmicos. São Carlos: Cubo, 2019. Disponível em: <https://www.bpbes.net.br/wp-content/uploads/2019/09/BPBES_ Completo_VF-1.pdf>. Acesso em: 15 dez. 2019.

Le Saout, S.; Hoffmann, M.; Shi, Y.; Hughes, A.; Bernard, C.; Brooks, T. M.; Bertzky, B.; Butchart, S. H. M.; Stuart, S. N.; Badman, T.; Rodrigues, A. S. L. Protected areas and effective biodiversity conservation. Science, v. 342, n.6160, p.803-805, 2013. https://doi.org/10.1126/science.1239268

Leung, Y.-F.; Spenceley, A.; Hvenegaard, G.; Buckley, R. Tourism and visitor management in protected areas: Guidelines for sustainability. Gland, Switzerland: IUCN, 2018. (Best practice protected area guidelines series, 27). https://doi.org/10.2305/IUCN.CH.2018. PAG.27.en

Leverington, F.; Costa, K. L.; Pavese, H.; Lisle, A.; Hockings, M. A global analysis of protected area management effectiveness. Environmental Management, v. 46, p. 685-698, 2010. https://doi.org/10.1007/s00267-010-9564-5 
Lima, E. A. C. F.; Ranieri, V. E. L. Land use planning around protected areas: Case studies in four state parks in the Atlantic forest region of Southeastern Brazil. Land Use Policy, v. 71, p. 453-458, 2018. https://doi.org/10.1016/j.landusepol.2017.12.020

Machado, R. X; Costa, E. A. O turismo de pesca em Corumbá, na fronteira Oeste do Brasil. Revista Iberoamericana de Turismo, v. 8, n. 1, p. 36-48, 2018.

Magro, T. C.; Watson, A.; Bernasconi, P. Identifying threats, values, and attributes in Brazilian wilderness areas. USDA Forest Service Proceedings RMRS-P-49, p. 319-322, 2007. Disponível em: <https://www.fs.usda.gov/treesearch/pubs/31048>. Acesso em: 15 jan. 2019.

NPS - National Park Service. 2019. Foundation document. Disponível em: <https://parkplanning.nps.gov/foundationDocuments.cfm>. Acesso em: 15 jan. 2019.

O'Donnel, T. Contrasting land use policies for climate change adaptation: A case study of political and geo-legal realities for Australian coastal locations. Land Use Policy, v. 88, p. 104-145 2019. https://doi.org/10.1016/j.landusepol.2019.104145

Peirce, C. S. Escritos coligidos. São Paulo: Nova Cultural, 1989.

Peñuelas, J.; Sardans, J.; Filella, I.; Estiarte, M.; Llusià, J.; Ogaya, R.; Carnicer, J.; Bartrons, M.; Rivas-Ubach, A.; Grau, O.; Peguero, G.; Margalef, M.; Pla-Rabés, S.; Stefanescu, C.; Asensio, D.; Preece, C.; Liu, L.; Verger, A.; Rico, L.; Barbeta, A.; Achotegui-Castells, A.; Gargallo-Garriga, A.; Sperlich, D.; Farré-Armengol, G.; Fernández-Martínez, M.; Liu, D.; Zhang, C.; Urbina, I.; Camino, M.; Vives, M.; Nadal-Sala, D.; Sabaté, S.; Gracia, C.; Terradas, J. Assessment of the impacts of climate change on Mediterranean terrestrial ecosystems based on data from field experiments and long-term monitored field gradients in Catalonia. Environmental and Experimental Botany, v.252, p.49-59, 2018. https://doi.org/10.1016/j.envexpbot.2017.05.012

Raulet-Croset, N. La dimension territoriale des situations de gestion. Revue Française de Gestion, v. 184, n. 4, p. 137-150, 2008. https://doi.org/10.3166/rfg.184.137-150

Rogora, M.; Frate, L.; Carranza, M. L.; Freppaz, M.; Stanisci, A.; Bertani, I.; Bottarin, R.; Brambilla, A.; Canullo, R.; Carbognani, M.; Cerrato, C.; Chelli, S.; Cremonese, E.; Cutini, M.; Di Musciano, M.; Erschbamer, B.; Godone, D.; Iocchi, M.; Isabellon, M.; Magnani, A.; Mazzola, L.; Morra di Cella, U.; Pauli, H.; Petey, M.; Petriccione, B.; Porro, F.; Psenner, R.; Rossetti, G.; Scotti, A.; Sommaruga, R.; Tappeiner, U.; Theurillat, J.-P.; Tomaselli, M.; Viglietti, D.; Viterbi, R.; Vittoz, P.; Winkler, M.; Matteucci, G. Assessment of climate change effects on mountain ecosystems through a cross-site analysis in the Alps and Apennines. Science of Total Environment, $\quad$ v. $624, \quad$ p. $1429-1442,2018 . \quad$ https://doi.org/10.1016/j.scitotenv. 2017.12.155

Suopajärvia, L.; Kantola, A. The social impact management plan as a tool for local planning: Case study: Mining in Northern Finland. Land Use Policy, v. 83, 2020. https://doi.org/10.1016/j.landusepol.2019.104046

Wang, J.-H. Z. National parks in China: Parks for people or for the nation? Land Use Policy, v. 85, p. 825-833, 2019. https://doi.org/10.1016/j.landusepol.2018.10.034

Informação da Licença: Este é um artigo Open Access distribuído sob os termos da Licença Creative Commons Attribution, que permite uso irrestrito, distribuição e reprodução em qualquer meio, desde que a obra original seja devidamente citada. 
Apêndice 1. Questionário enviado online para profissionais-chave.

\section{Plano de manejo (PM): Modelo Tradicional x Foundation Document}

A elaboração de Planos de Manejo (PMs) é uma obrigação legal, constante da Lei no 9.985/2000 (SNUC). Gostaríamos de saber, a partir da sua experiência, sua opinião quanto ao uso do Foundation Document para a elaboração de PMs. São, no máximo, sete perguntas, cujo tempo estimado de resposta é de cinco minutos. Desde já, agradecemos pela sua participação!

1. Ao responder este questionário, você concorda que utilizemos a informação sem citar a fonte?

( ) Sim.

( ) Não.

2. Há quantos anos trabalha com a elaboração de Planos de Manejo? (campo: número > 0).

3. Já participou ou está participando da elaboração de algum Plano de Manejo com o Foundation? Document?
( ) Sim.
( ) Não.

4. Em quantos Planos de Manejo no formato Foundation você participou (contando os que estão em andamento)? (campo: número $>0$ ).

5. Comparando o Roteiro Metodológico de Planejamento, anteriormente usado, com o Foundation Document, você vê mais vantagens ou desvantagens nesse último e em quais itens?

Respostas:

( ) Mais desvantagens.

( ) Igual.

( ) Mais vantagens.

Itens:

( ) Não sei opinar.
( ) Número de servidores envolvidos.
( ) Custo total de elaboração.
( ) Tempo de elaboração.
( ) Participação social.
( ) Necessidade de produção de documentos futuros.
( ) Facilidade de revisão.
( ) Atingimento dos objetivos das Unidades de Conservação (UCs).

6. Quanto à elaboração de Planos de Manejo pelo Roteiro Metodológico de Planejamento, você o considera:

Respostas:
( ) Baixo.
( ) Moderado.
( ) Alto.
( ) Não sei opinar.

Itens:
( ) Tem um custo financeiro.
( ) Envolvimento da sociedade civil.
( ) 0 custo de revisão é.
( ) Qual o grau de atendimento aos objetivos das UCs.

7. Você destacaria outros métodos para a elaboração de Planos de Manejo? Quais? (Resposta aberta - textual).

8. Você gostaria de acrescentar e/ou comentar alguma outra informação que não foi apresentada aqui? (Resposta aberta - textual). 